\title{
The impact of continuous positive airway pressure on radiation dose to heart and lung during left-sided postmastectomy radiotherapy when deep inspiration breath hold technique is not applicable: a case report
}

\author{
Whoon Jong Kil, MD', Tabitha Pham, CMD1, Sabbir Hossain, PhD', Juan Casaigne, CMD', \\ Kellie Jones, MD ${ }^{1,2}$, Mohammad Khalil, MD ${ }^{1,2}$ \\ 'Medicine Service, Radiation Oncology Clinic, Oklahoma City VA Health Care System, Oklahoma City, OK; \\ ${ }^{2}$ University of Oklahoma Health Science Center, Oklahoma City, OK, USA
}

Deep inspiration breathing hold (DIBH) compared to free-breathing (FB) during radiotherapy (RT) has significantly decreased radiation dose to heart and has been one of the techniques adopted for patients with breast cancer. However, patients who are unable to make suitable deep inspiration breath may not be eligible for DIBH, yet still need to spare the heart and lung during breast cancer RT (left-sided RT in particular). Continuous positive airway pressure (CPAP) is a positive airway pressure ventilator, which keeps the airways continuously open and subsequently inflates the thorax resembling thoracic changes from DIBH. In this report, authors applied CPAP instead of FB during left-sided breast cancer RT including internal mammary node in a patient who was unable to tolerate DIBH, and substantially decreased radiation dose the heart and lung with CPAP compared to FB.

Keywords: Breast cancer, Left-sided PMRT, Heart, Lung, Continuous positive airway pressure (CPAP)

\section{Introduction}

Postmastectomy radiotherapy (PMRT) to chest wall (CW) and regional nodal stations (RNS; e.g., axillary, supraclavicular, and internal mammary [IMN] nodal stations) for patients with node-positive breast cancer has been shown to lower the risk of locoregional and distant recurrences, improve disease free survival and overall survival [1-3]. PMRT including RNS, however, can increase radiation dose to the heart and lung. Although other risk factors (i.e., hypercholesterolemia, hypertension, and cigarette smoking) are also closely related with cardiac and pulmonary events, it has been emphasized to lower radiation dose to the heart and lung as low as reasonably achievable during radiotherapy (RT) for breast cancer to reduce RT-related cardiac and pulmonary toxicities [4-6].

Deep inspiration breathing hold (DIBH) compared to freebreathing (FB) during RT has significantly decreased radiation dose to heart and has been one of the techniques adopted for patients with breast cancer $[7,8]$. Despite these dosimetric benefits, however, patients who cannot maintain and/or reproduce suitable deep breath may not be eligible for DIBH during RT for breast cancer [9].

Continuous positive airway pressure (CPAP) is a positive

Received 06 January 2018, Revised 25 January 2018, Accepted 02 February 2018.

Correspondence: Whoon Jong Kil, MD, Medicine Service, Radiation Oncology Clinic, Oklahoma City Veterans Affairs Health Care System, Oklahoma City, OK, USA. Tel: +1-405-456-4450, Fax: +1-405-456-4472, E-mail: whoonkil@gmail.com

(c) This is an Open Access article distributed under the terms of the Creative Commons Attribution Non-Commercial License (http://creativecommons.org/ licenses/by-nc/4.0/) which permits unrestricted non-commercial use, distribution, and reproduction in any medium, provided the original work is properly cited.

www.e-roj.org 
airway pressure ventilator applying mild air pressure on a continuous basis to keep the airways continuously open and subsequently the thorax will be more inflated in comparison to FB. Physical changes in thorax with CPAP can potentially affect the radiation dose to the heart and lung during $\mathrm{RT}$ for breast cancer.

Herein, authors report decreased radiation doses to the heart and lung with CPAP compared to FB during left-sided PMRT including RNS in a patient who was unable to maintain and reproduce suitable DIBH.

\section{Case Report}

\section{Case presentation}

A 53-year-old female with a family history of breast cancer (paternal and maternal grandmothers) and significant tobacco abuse (one half to one full pack of cigarettes per day since 9 years old) noticed a lump in the left breast. On physical examination, there was a $10 \mathrm{~cm} \times 5 \mathrm{~cm}$ palpable mass in the left breast and a palpable deep left axillary lymph node. Her right breast was notable for nipple retraction with a $2 \mathrm{~cm} \times 4 \mathrm{~cm}$ palpable mass but no axillary lymphadenopathy. Mammogram, breast magnetic resonance imaging scan, and positron emission tomography-computed tomography (PET-CT) scan demonstrated a $3.1-\mathrm{cm}$ mass in the right breast at 12 o'clock, a $2.8-\mathrm{cm}$ mass with satellite mass in the left breast at 2 o'clock, and axillary lymphadenopathy. Biopsy noted an invasive ductal carcinoma (IDC), estrogen/progesterone receptor weakly positive approximately 3\%, human epidermal growth factor receptor 2 (HER2) positive staining intensity 3+,50\% Ki-67 at the left breast mass and axilla node, and ductal hyperplasia at the right breast lesion. After length of discussion with the surgeon, she elected to pursue left mastectomy, left axillary nodes dissection, and prophylactic right mastectomy. Pathology reported three foci of IDC (maximum $2.5 \mathrm{~cm}$, grade 2 ) in the lower outer quadrant and ductal carcinoma in situ (DCIS) with focal microinvasion in the left breast; metastatic IDC in three (largest $1.4 \mathrm{~cm}$ ) out of nine axillary lymph nodes with extracapsular extension. Negative surgical resection margin ( $1 \mathrm{~cm}$ from both IDC and DCIS) was obtained. Right breast specimen showed fibrocystic changes. Genetic testing for breast cancer gene (BRCA) mutation was negative. She completed adjuvant chemotherapy with 4 cycles of Adriamycin and cyclophosphamide followed by 4 cycles of paclitaxel and Herceptin. Subsequently, she continued Herceptin treatments for a total of one year when she was referred to the radiation oncology clinic for PMRT.
After consultation with a radiation oncologist, she was recommended a left-sided PMRT including RNS to reduce the risks of local-regional cancer recurrence. During breathing education for DIBH, however, she developed shortness of breath quickly after few short-shallow breaths and could not reproduce DIBH. Since she has used CPAP to prevent airway obstruction during sleep for years, the radiation oncologist suggested that she wears CPAP during PMRT rather than FB because continuous positive airway pressure could potentially keep inflating the thorax similar to DIBH effects on the thorax. Before computed tomography (CT)-simulation, she visited the CPAP clinic to find the maximum tolerable airway pressure between 6 to $15 \mathrm{~cm} \mathrm{H}_{2} \mathrm{O}$ with 0.21 of fraction of inspired oxygen (12 $\mathrm{cm} \mathrm{H}_{2} \mathrm{O}$ in her case), and reassure if the CPAP face mask was well fitted on her face. Radiation therapists were educated by respiratory therapists for set-up CPAP machine during CT-simulation and daily treatment. First, she underwent CT-simulation with FB (CT-FB) followed by 30 minutes of using CPAP with $12 \mathrm{~cm} \mathrm{H}_{2} \mathrm{O}$. Second, a repeat $\mathrm{CT}$-simulation was done with CPAP (CT-CPAP) on the same day with same position between scans.

Planning target volumes (PTVs; CW and RNS) and organs at risk (OARs) were contoured on both CT-CPAP and CT-FB scans for the pretreatment RT planning according to RTOG 1304. Prescription was 50 Gy to PTVs in 25 fractions. No boost was given. Treatment planning aimed to reduce doses to OARs as much as possible without compromising the coverage of the PTVs. Eclipse RT planning system version 10.0 (Varian Medical System, Palo Alto, CA, USA) was used with a Varian Trilogy linear accelerator using 6-MV photons. First, pretreatment PMRT plans were created using three-dimensional conformal radiotherapy (3DCRT). Subsequent volumetric modulated arc therapy (VMAT) was planned to see any dosimetric benefits over 3DCRT in this patient. The 4-field 3DCRT photon only plan consisted of two tangential fields covering PTV-CW and IMN, and anterior-posterior fields covering other PTV-RNS. Due to the technical limitation at our clinic, photon-electron match technique was not pursued after the initial attempt on CT-CPAP failing to achieve safe and efficient RT plan. The beam's-eye view was used to shape multi-leaf collimators to block OARs. Field-in-field technique was used to maximize dose homogeneity. VMAT planning consisted of four $200^{\circ}$ partial arcs with $2 \mathrm{~cm}$ overlapping jaws (two clockwise and two counterclockwise; gantry angle $300^{\circ}-140^{\circ}$ ) to completely encompass PTVs with involved supraclavicular and IMN with $30^{\circ}$ or $330^{\circ}$ collimation angles. Plan quality was determined by PTV and PTV_evaluation (PTVeval) structures coverage and 
OARs dose according to RTOG 1304.

\section{Case results}

1) Physical changes in thorax with CPAP

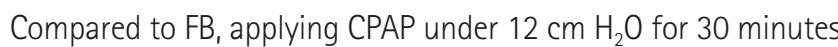
to the patient inflated the thorax and increased the lung volumes (Table 1, Fig. 1). Left lung volume was $1,189.99 \mathrm{~cm}^{3}$ with $\mathrm{FB}$ and $1,552.01 \mathrm{~cm}^{3}$ with CPAP (30.4\% increase). Total lung volume was also increased by $748.22 \mathrm{~cm}^{3}$ with CPAP in comparison to FB. The inflated thorax with CPAP displaced the heart more inferiorly than with FB. The distance from sternal notch to the superior portion of heart contour was $6.2 \mathrm{~cm}$
(CPAP) and $5.25 \mathrm{~cm}$ (FB). With CPAP, the heart was physically more separated from left CW than with FB. The heart volume within the tangent RT field was $35.48 \mathrm{~cm}^{3}$ with $\mathrm{FB}$ to $2.79 \mathrm{~cm}^{3}$ with CPAP (92.2\% reduction).

\section{2) Radiation target coverages}

Coverage for PMRT plans with FB+3DCRT, CPAP+3DCRT, $\mathrm{FB}+\mathrm{VMAT}$, and CPAP+VMAT are displayed in Table 2. All PMRT plans met acceptable PTV and PTVeval structures coverage per RTOG 1304. VMAT demonstrated better PTVeval-CW receiving $\geq 45$ Gy $\left(V_{45}, 95 \%\right.$ with both CPAP and FB) than 3DCRT $(92 \%$ and $91 \%$ with CPAP and FB, respectively).

\section{Free-Breathing}
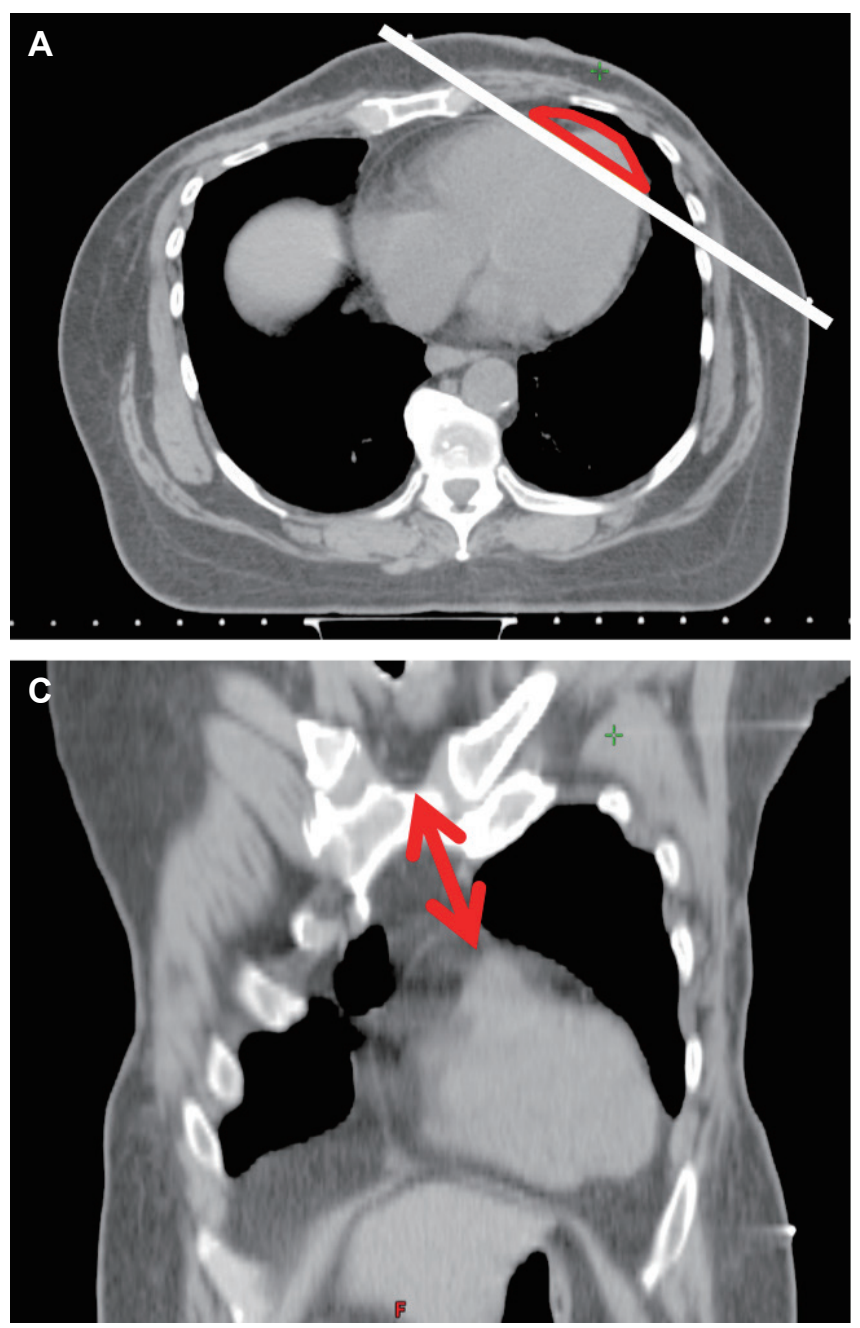

\section{CPAP}
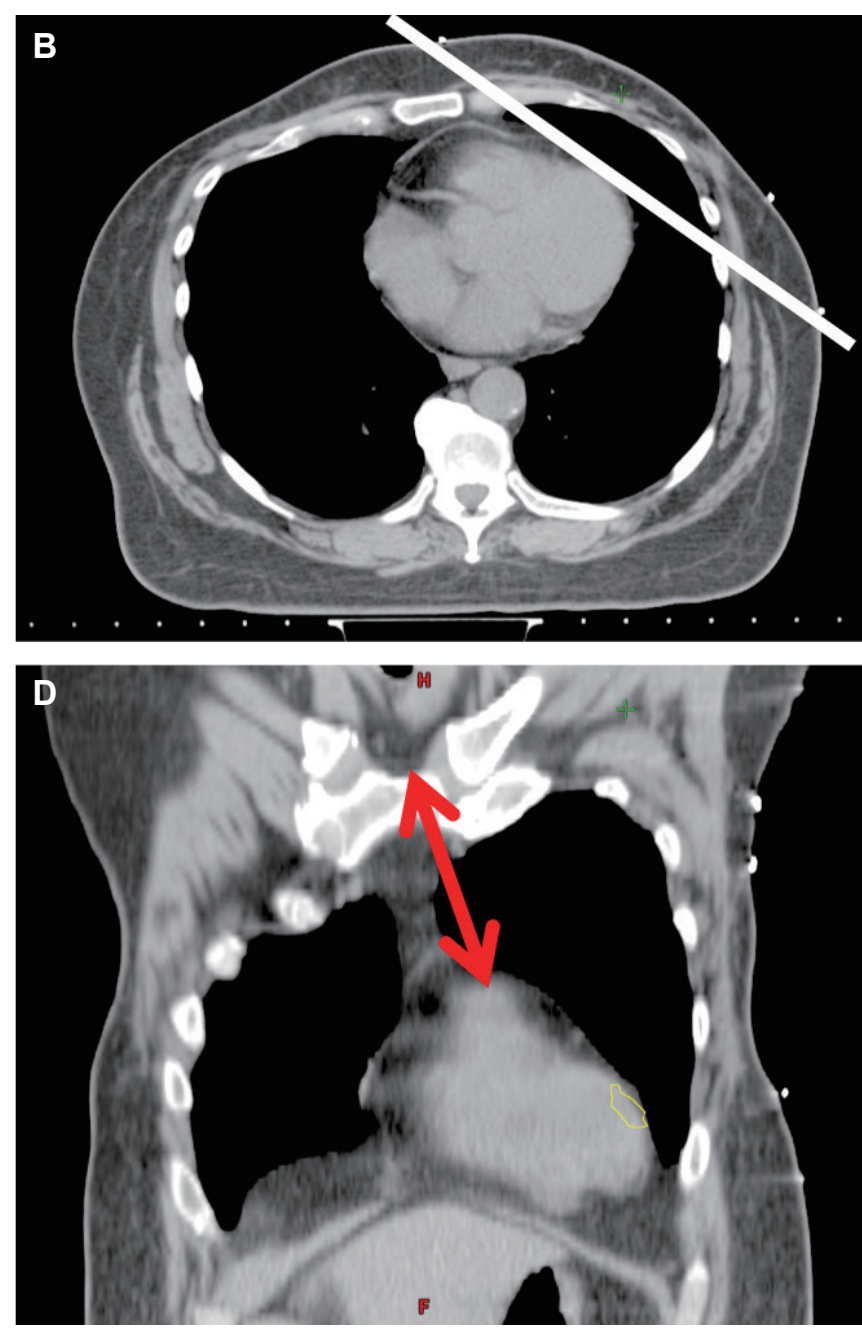

Fig. 1. Physical changes in the thorax between free-breathing (FB) and continuous positive airway pressure (CPAP; $12 \mathrm{~cm} \mathrm{H}_{2} \mathrm{O}$ for 30 minutes before and during scan). Axial image on computed tomography-simulation scan with FB (A) and with CPAP (B), and coronal image with FB (C) and with CPAP (D). White solid line in (A) and (B) indicates tangent radiotherapy field at the same level; red contour in $(A)$, the heart within tangent radiotherapy field; red arrow in (C) and (B), the distance from sternal notch to the superior part of heart contour. 
Table 1. Physical changes in thorax with CPAP

\begin{tabular}{|c|c|c|c|c|c|}
\hline & \multicolumn{3}{|c|}{ Lung volume $\left(\mathrm{cm}^{3}\right)$} & \multicolumn{2}{|l|}{ Heart } \\
\hline & Left & Right & Total & Within tangent field $\left(\mathrm{cm}^{3}\right)$ & From sternal notch $(\mathrm{cm})$ \\
\hline$F B$ & $1,189.99$ & $1,389.86$ & $2,579.87$ & 35.48 & 5.25 \\
\hline CPAP & $1,552.01$ & $1,776.08$ & $3,328.09$ & 2.76 & 6.50 \\
\hline Difference & +362.02 & +386.22 & +748.22 & -32.72 & +1.25 \\
\hline
\end{tabular}

CPAP, continuous positive airway pressure; FB, free-breathing; Difference, changes from FB to CPAP; '+', increase; '-', reduction; Within tangent field, heart volume within tangent radiotherapy field; From sternal notch, distance from sternal notch to superior portion of heart contour.

Table 2. Radiation target coverages

\begin{tabular}{|c|c|c|c|c|c|c|c|c|c|}
\hline & & \multicolumn{8}{|c|}{ PTV (\%) } \\
\hline & & \multicolumn{2}{|c|}{ CWeval } & \multicolumn{2}{|c|}{$A X$} & \multicolumn{2}{|c|}{$\mathrm{SCL}$} & \multicolumn{2}{|c|}{ IMN } \\
\hline & & $V_{47.5}$ & $V_{45}$ & $V_{47.5}$ & $\mathrm{~V}_{45}$ & $V_{47.5}$ & $V_{45}$ & $V_{47.5}$ & $V_{45}$ \\
\hline \multirow[t]{2}{*}{ 3DCRT } & FB & 88 & 91 & 96 & 98 & 90 & 94 & 79 & 100 \\
\hline & CPAP & 88 & 92 & 91 & 98 & 91 & 95 & 75 & 100 \\
\hline \multirow[t]{2}{*}{ VMAT } & FB & 81 & 95 & 77 & 95 & 72 & 93 & 87 & 97 \\
\hline & CPAP & 78 & 95 & 65 & 95 & 60 & 91 & 84 & 100 \\
\hline
\end{tabular}

PTV, planning target volume; CWeval, chest wall_evaluation; AX, axillary nodal station; SCL, supraclavicular nodal station; IMN, internal mammary nodal station; $V_{47.5}, V_{45}$ and $V_{40}$, volume receiving $\geq 47.5,45$, and $40 \mathrm{~Gy}$, respectively; 3DCRT, three-dimensional conformal radiotherapy; VMAT, volumetric modulated arc therapy; FB, free-breathing; CPAP, continuous positive airway pressure.

Table 3. Radiation dose to organs at risk

\begin{tabular}{|c|c|c|c|c|c|c|c|c|c|c|}
\hline & & \multicolumn{3}{|c|}{ Heart } & \multicolumn{4}{|c|}{ Lung-left (ipsilateral) } & \multicolumn{2}{|c|}{ Lung-right } \\
\hline & & $\mathrm{D}_{\text {mean }}(\mathrm{Gy})$ & $\mathrm{V}_{30}(\%)$ & $\mathrm{V}_{25}(\%)$ & $D_{\text {mean }}(G y)$ & $V_{20}(\%)$ & $\mathrm{V}_{10}(\%)$ & $V_{5}(\%)$ & $\mathrm{D}_{\text {mean }}(\mathrm{Gy})$ & $V_{5}(\%)$ \\
\hline \multirow[t]{2}{*}{ 3DCRT } & $F B$ & 11 & 16 & 18 & 19 & 39 & 48 & 56 & 0.3 & 0 \\
\hline & CPAP & 3 & 2 & 2 & 17 & 36 & 44 & 54 & 0.3 & 0 \\
\hline \multirow[t]{2}{*}{ VMAT } & $\mathrm{FB}$ & 6 & 0.6 & 2 & 15 & 29 & 57 & 81 & 4 & 15 \\
\hline & CPAP & 5 & 0 & 0.1 & 14 & 24 & 54 & 75 & 3 & 10 \\
\hline
\end{tabular}

$D_{\text {mean, }}$ mean dose; $V_{30}, V_{25}, V_{20}, V_{10}$, and $V_{5}$, volume receiving $\geq 30,25,20,10$, and 5 Gy, respectively; 3DCRT, three-dimentional conformal radiotherapy; VMAT, volumetric modulated arc therapy; FB, free-breathing; CPAP, continuous positive airway pressure.

\section{3) Radiation dose to OARs}

Radiation dose to the heart was less with CPAP than FB (Table 3). Mean dose $\left(D_{\text {mean }}\right)$ to the heart was $3 \mathrm{~Gy}(C P A P+3 D C R T)$, 5 Gy (CPAP+VMAT), 6 Gy (FB+VMAT), and 11 Gy (FB+3DCRT). Although only PMRT plan with CPAP+3DCRT met $D_{\text {mean }}$ to the heart, CPAP+VMAT also showed acceptable $D_{\text {mean }}$ to the heart (5 Gy). For $V_{25}$ to the heart, PMRT plan with CPAP+VMAT demonstrated the lowest percentage $(0.1 \%)$ in comparison to the others $(2 \%, 2 \%$, and $18 \%$ with FB+VMAT, CPAP+3DCRT, and $\mathrm{FB}+3 \mathrm{DCRT}$, respectively).

PMRT plans with VMAT spared radiation dose to ipsilateral lung compared with 3DCRT. Dmean to ipsilateral lung was 14 Gy (CPAP+VMAT), 15 Gy (FB+VMAT), 17 Gy (CPAP+3DCRT), and $19 \mathrm{~Gy}(\mathrm{FB}+3 \mathrm{DCRT})$. Only PMRT plans with VMAT met $V_{20}$ to ipsilateral lung (24\% with CPAP+VMAT and 29\% with
FB+VMAT). PMRT plans with 3DCRT, however, failed to meet $V_{20}$ to ipsilateral lung (36\% with CPAP+3DCRT and 39\% with $\mathrm{FB}+3 \mathrm{DCRT})$. Although VMAT delivered more radiation dose to right lung, $V_{5}$ to contralateral lung was acceptable with CPAP+VMAT (10\%). Table 3 lists radiation doses to the OARs in PMRT plans.

\section{Discussion}

Radiation dose to the heart and lung during RT for breast cancer has been reported to be related with cardiac and pulmonary toxicities [4-6]. Data suggest that the rate of major coronary event increases linearly with mean heart dose by 7.4\% per Gy, with higher absolute risks among individuals with preexisting cardiac risks [5]. Since there is no known radiation 
dose threshold for cardiac toxicity, it becomes extremely important to limit cardiac dose during RT for breast cancer. The incidence of radiation pneumonitis is also well correlated with mean lung dose and lung $V_{20}$ [6]. With modern RT for breast cancer, estimated absolute risks of lung cancer were approximately 4\% for long-term continuing smokers and $0.3 \%$ for non-smoker; cardiac mortality was 1\% for smoker and $0.3 \%$ for non-smokers [4]. Although the absolute risk of these cardiac and pulmonary toxicities after RT for breast cancer can be considered low and confounded by other variables (i.e., smoking history), all possible efforts to reduce these risks as much as reasonably achievable should be taken, since the majority of patients will be long-term survivors and at risk of late toxicity after RT for breast cancer.

Among the various RT techniques for patients with breast cancer, DIBH has demonstrated the ability to inflate the thorax and displace the heart inferiorly resulting in increased lung volume and separation between the heart and CW. These physical changes in the thorax with DIBH have shown less radiation dose to the heart and lung than with FB [79]. Despite these benefits, however, suitable and reproducible $\mathrm{DIBH}$ is highly dependent on the patients breathing habit, lung function, and compliance to the breathing education for DIBH. Patients with shallow breathing or chronic obstructive pulmonary diseases might not be able to reproduce and maintain deep enough inspiration during RT for breast cancer. There are also concerns about technical complexity and infrastructural constraints such as appropriate equipment to monitor, detect, and deliver RT only during deep breath [10]. Experienced radiation oncologist and staff are required for successfully and safely implementing the DIBH technique.

CPAP has been widely used for patients with obstructive sleep apnea. Although there is no data reporting the occurrence of CPAP usage in breast cancer patients, estimated prevalence of obstructive sleep apnea is in the range of $3 \%$ to $7 \%$ among general population [11]. In this report, authors noticed that CPAP inflated the thorax, displaced the heart inferiorly, and decreased the heart volume within tangent RT field resembling physical changes seen in the thorax from DIBH (Table 1, Fig. 1). These physical changes with CPAP decreased radiation dose to the heart and lung during leftsided PMRT including RNS (Table 3). The findings in this report suggest that CPAP instead of FB can be of benefits for breast cancer patients who were unable to make suitable DIBH during RT.

Comparing RT techniques, PMRT with CPAP+VMAT provided comparable PTV and PTVeval structures coverages with the lowest or meeting acceptable radiation dose constraints for the heart and lungs than with 3DCRT in this patient. Thus, the patient received PMRT including RNS with CPAP+VMAT Given her history of cigarette smoking and continued smoking during the course of PMRT, decreased radiation dose to the heart and lung with CPAP+VMAT might translate into reduced cardiac and pulmonary toxicities.

In conclusion, for a patient who needed left-sided PMRT including RNS but was unable to reproduce and maintain deep inspiration, using CPAP during PMRT showed physical changes in the thorax similarly to DIBH resulting in decreased radiation dose to the heart and lung. These findings suggest that using CPAP during RT for breast cancer could be considered as one of alternative methods to minimize radiation dose to the heart and lung when DIBH is not applicable.

\section{Conflict of Interest}

No potential conflict of interest relevant to this article was reported.

\section{References}

1. Thorsen LB, Offersen BV, Dano H, et al. DBCG-IMN: a population-based cohort study on the effect of internal mammary node irradiation in early node-positive breast cancer. J Clin Oncol 2016;34:314-20.

2. EBCTCG (Early Breast Cancer Trialists' Collaborative Group), McGale $\mathrm{P}$, Taylor $\mathrm{C}$, et al. Effect of radiotherapy after mastectomy and axillary surgery on 10-year recurrence and 20-year breast cancer mortality: meta-analysis of individual patient data for 8135 women in 22 randomised trials. Lancet 2014;383:2127-35.

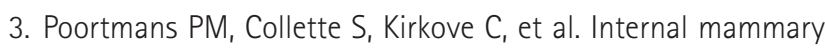
and medial supraclavicular irradiation in breast cancer. $N$ Engl J Med 2015;373:317-27.

4. Taylor C, Correa C, Duane FK, et al. Estimating the risks of breast cancer radiotherapy: evidence from modern radiation doses to the lungs and heart and from previous randomized trials. J Clin Oncol 2017;35:1641-9.

5. Darby SC, Ewertz M, Hall P. Ischemic heart disease after breast cancer radiotherapy. N Engl J Med 2013;368:2527.

6. Marks LB, Yorke ED, Jackson A, et al. Use of normal tissue complication probability models in the clinic. Int J Radiat Oncol Biol Phys 2010;76(3 Suppl):S10-9.

7. Zagar TM, Kaidar-Person 0 , Tang $X_{1}$ et al. Utility of deep inspiration breath hold for left-sided breast radiation therapy 
Whoon Jong Kil, et al

in preventing early cardiac perfusion defects: a prospective study. Int J Radiat Oncol Biol Phys 2017;97:903-9.

8. Conway $\mathrm{L}$, Conroy L, Harper L, et al. Deep inspiration breathhold produces a clinically meaningful reduction in ipsilatera lung dose during locoregional radiation therapy for some women with right-sided breast cancer. Pract Radiat Oncol 2017;7:147-53.

9. Rice L, Goldsmith C, Green MM, Cleator S, Price PM. An effective deep-inspiration breath-hold radiotherapy technique for left-breast cancer: impact of post-mastectomy treatment, nodal coverage, and dose schedule on organs at risk. Breast Cancer (Dove Med Press) 2017;9:437-46.

10. Boda-Heggemann J, Knopf AC, Simeonova-Chergou $A$, et al. Deep inspiration breath hold-based radiation therapy: a clinical review. Int J Radiat Oncol Biol Phys 2016;94:478-92.

11. Punjabi NM. The epidemiology of adult obstructive sleep apnea. Proc Am Thorac Soc 2008;5:136-43. 